Check for updates

Cite this: RSC Adv., 2019, 9, 19325

Received 1st May 2019

Accepted 10th June 2019

DOI: 10.1039/c9ra03251g

rsc.li/rsc-advances

\section{Validated liquid chromatography tandem mass spectrometry for simultaneous quantification of foretinib and lapatinib, and application to metabolic stability investigation}

\author{
Mohammed M. Alanazi, ${ }^{a}$ Hamad M. Alkahtani, (DD a Abdulrahman A. Almehizia, (DD *a \\ Mohamed W. Attwa, (D) *ab Ahmed H. Bakheit ${ }^{\text {ac }}$ and Hany W. Darwish ${ }^{\text {ad }}$
}

Foretinib (GSK1363089, FTB) is a multikinase inhibitor that inhibits multiple receptor tyrosine kinases, including vascular endothelial growth factor receptor-2 and mesenchymal-epithelial transition factor, with the potential for solid tumor treatment. Lapatinib (LPB) is a significant promising drug molecule that was approved by the USFDA and was utilized to develop a nontoxic and very efficient targeted therapy against breast cancer. There is an ongoing clinical trial for using of FTB and LPB combination for HER-2 positive metastatic breast cancer treatment. In the current study, liquid chromatography tandem mass spectrometry methodology was validated for simultaneous estimation of FTB and LPB with application to drug metabolic stability investigation. Chromatographic separation of FTB, LPB and masitinib (internal standard) was attained using an isocratic mobile phase running on a reversed-phase $\mathrm{C}_{18}$ column. The linear dynamic range was $5-500 \mathrm{ng} \mathrm{mL}^{-1}$ with $r^{2} \geq 0.9999$ in the rat liver microsomes (RLMs) matrix. The FTB and LPB metabolic stabilities in the RLMs matrix were estimated by computing two parameters, intrinsic clearance $\left(\mathrm{CL}_{\text {int }}: 6.33\right.$ and $5.63 \mathrm{~mL} \mathrm{~min}^{-1} \mathrm{~kg}^{-1}$ ) and a low in vitro half-life $\left(t_{1 / 2}: 23.9\right.$ and 26.9 $\mathrm{min})$, which revealed the FTB and LPB high clearance by the liver from the blood. This probably revealed the low in vivo bioavailability that verified the low oral bioavailability previously reported and also indicated that FTB and LPB will not bioaccumulate after multiple doses. FTB metabolic rate is slightly decreased in combination with LPB, while LPB metabolic rate is greatly increased in combination with FTB. So dose recalculation must be evaluated when FTB and LPB are used in combination.

\section{Introduction}

Tyrosine kinase inhibitors (TKIs) are a class of drugs that inhibit the intracellular signals that drive proliferation in numerous malignant cells by unambiguously inhibiting the kinase activities of distinct intracellular pathways involved in receptor mediated growth signaling. ${ }^{\mathbf{1 , 2}}$ Tyrosine kinases are enzymes which control $\gamma$ phosphate group of ATP transfer to the tyrosine hydroxyl groups on marked proteins. They play as "on" or "off" switch in various cellular functions. ${ }^{3}$ Firm management of the tyrosine kinase activity in the cell controls important processes such as proliferation, cell cycle and cell death. In several cases, the cancer abnormal proliferation characteristics

${ }^{a}$ Department of Pharmaceutical Chemistry, College of Pharmacy, King Saud University, P.O. Box 2457, Riyadh, 11451, Saudi Arabia.E-mail: mehizia@ksu.edu.sa; mzeidan@ ksu.edu.sa; Fax: +966 114676 220; Tel: +966 114670237

${ }^{b}$ Students' University Hospital, Mansoura University, Mansoura 35516, Egypt ${ }^{c}$ Department of Chemistry, Faculty of Science and Technology, Al-Neelain University, Khartoum, Sudan

${ }^{d}$ Analytical Chemistry Department, Faculty of Pharmacy, Cairo University, Kasr El-Aini St., Cairo 11562, Egypt are generated by growth factor receptor-mediated signaling. In tumor cells, the failure of the control mechanism may initiate excessive phosphorylation, and pathways retained in an activated state. ${ }^{4,5}$ Foretinib (FTB) and lapatinib (LPB) are TKIs.

FTB (GSK1363089, Fig. 1) is a multikinase inhibitor that inhibits multiple receptor tyrosine kinases, including vascular endothelial growth factor receptor-2 (VEGFR) and mesenchymal-epithelial transition factor (MET), with the capability for solid tumors treatment. ${ }^{6}$ GlaxoSmithKline launched many clinical trials for FTB in many types of cancers but in 2015, it suddenly discontinued their clinical studies for FTB. LPB (Fig. 1) is a significant promising drug molecules, approved by FDA, USA is being utilizing for the development of a nontoxic and effective targeted therapy against breast cancer. ${ }^{7}$ The LPB clinical effectiveness in combination with capecitabine has exhibited efficacy contrary to HER2-positive breast cancer. ${ }^{8}$ Besides, LPB-loaded human serum albumin nano particles have been proposed as a nontoxic therapy against HER2-positive cells. ${ }^{9}$ LPB belongs to a class of tyrosine kinase inhibitor, is hydrophobic in nature. LPB suppresses the abnormal activity of HER2 and EGFR by inhibiting their phosphorylation. ${ }^{10}$ Even 
<smiles>COc1cc2c(Oc3ccc(/N=C(\O)C4(C(=O)Nc5ccc(F)cc5)CC4)cc3F)ccnc2cc1OCCCN1CCOCC1</smiles>

Foretinib

Molecular Weight: 632.66<smiles>CS(=O)(=O)CCNCc1ccc(-c2ccc3ncnc(Nc4ccc(OCc5cccc(F)c5)c(Cl)c4)c3c2)o1</smiles>

Molecular Weight: 581.06<smiles>Cc1ccc(NC(=O)c2ccc(CN3CCN(C)CC3)cc2)cc1Nc1nc(-c2cccnc2)cs1</smiles>

\section{Masitinib (IS)}

Molecular Weight: 498.65

Fig. 1 Chemical structures of foretinib, lapatinib and masitinib (IS).

though, LPB has exhibited $99 \%$ bound to $\alpha-1$ acid glycoprotein and human serum albumin in the blood stream. ${ }^{11}$

There is an undergoing clinical trial for the use of FTB and LPB combination for the treatment of HER-2 positive metastatic breast cancer. ${ }^{12}$ To the best of our knowledge, there has been no published analytical method for simultaneous estimation of FTB and LPB. Therefore, the purpose of the present study was to establish a validated LC-MS/MS method to quantify FTB and
LPB with application to metabolic stability estimation by computing $\mathrm{CL}_{\text {int }}$ in vitro half-life $\left(t_{1 / 2}\right)$. These parameters could then be used for bioavailability calculations, hepatic clearance and in vivo $t_{1 / 2}$. Bioavailability is crucial as it provides an idea for tested drug metabolism; if the drug is rapidly metabolized, it will showed low bioavailability in vivo. ${ }^{13}$ Also studying the combined form metabolic stability may gave an idea about the drug-drug interaction between FTB and LPB.<smiles>CCCN1CCOCC1</smiles>

Scheme 1 Product ion of FTB (A), LPB (B) and MST (C). 
Table 1 Optimized parameters of LC-MS/MS method

Parameters of Acquity UPLC

Isocratic mobile phase

Foretinib (FTB)

IS

ACQUITY UPLC BEH C18 column at T: $2250 \mathrm{~mm}$ in length

$\pm 1{ }^{\circ} \mathrm{C}$

Analyte

${ }^{a}$ Cone voltage. ${ }^{b}$ Collision energy.

$\mathrm{H}_{2} \mathrm{O}$ formic acid))

$130 \AA$ Å pore size

Lapatinib (LPB)

Masitinib (MST)
Parameters of Acquity TQD MS

$45 \%$ ACN

$55 \%$ aqueous $(10 \mathrm{mM}$ ammonium formate in

(pH: 4.2 adjusted by adding few drops of

Flow rate: $0.25 \mathrm{~mL} \mathrm{~min}^{-1}$

Injection volume: $5 \mu \mathrm{L}$

$2.1 \mathrm{~mm}$ in internal diameter

$1.7 \mu \mathrm{m}$ particle size

Transitions

Flow rate $\left(12 \mathrm{~L} \mathrm{~min}^{-1}\right)$

Pressure (60 psi)

Source temperature: $350{ }^{\circ} \mathrm{C}$

Capillary voltage: $4000 \mathrm{~V}$

Collision cell gas Nitrogen with high purity

Mode

Multiple reaction monitoring (MRM)

LPB MRM

$m / z 581 \rightarrow m / z 350, \mathrm{CO}^{a}: 62 \mathrm{~V}, \mathrm{CE}^{b}:$ of $38 \mathrm{eV}$

$m / z 581 \rightarrow m / z 365, \mathrm{CO}^{a}: 62 \mathrm{~V}, \mathrm{CE}:$ of $38 \mathrm{eV}$

FTB MRM $\quad m / z 633 \rightarrow m / z 100, \mathrm{CO}^{a}: 46 \mathrm{~V}, \mathrm{CE}^{b}:$ of transitions $\quad 34 \mathrm{eV}$

$m / z 633 \rightarrow m / z 128, \mathrm{CO}^{a}: 46 \mathrm{~V}, \mathrm{CE}:$ of $32 \mathrm{eV}$

$m / z 499 \rightarrow m / z 399.06 \mathrm{CO}^{a}: 42 \mathrm{~V}, \mathrm{CE}:$ $24 \mathrm{eV}$

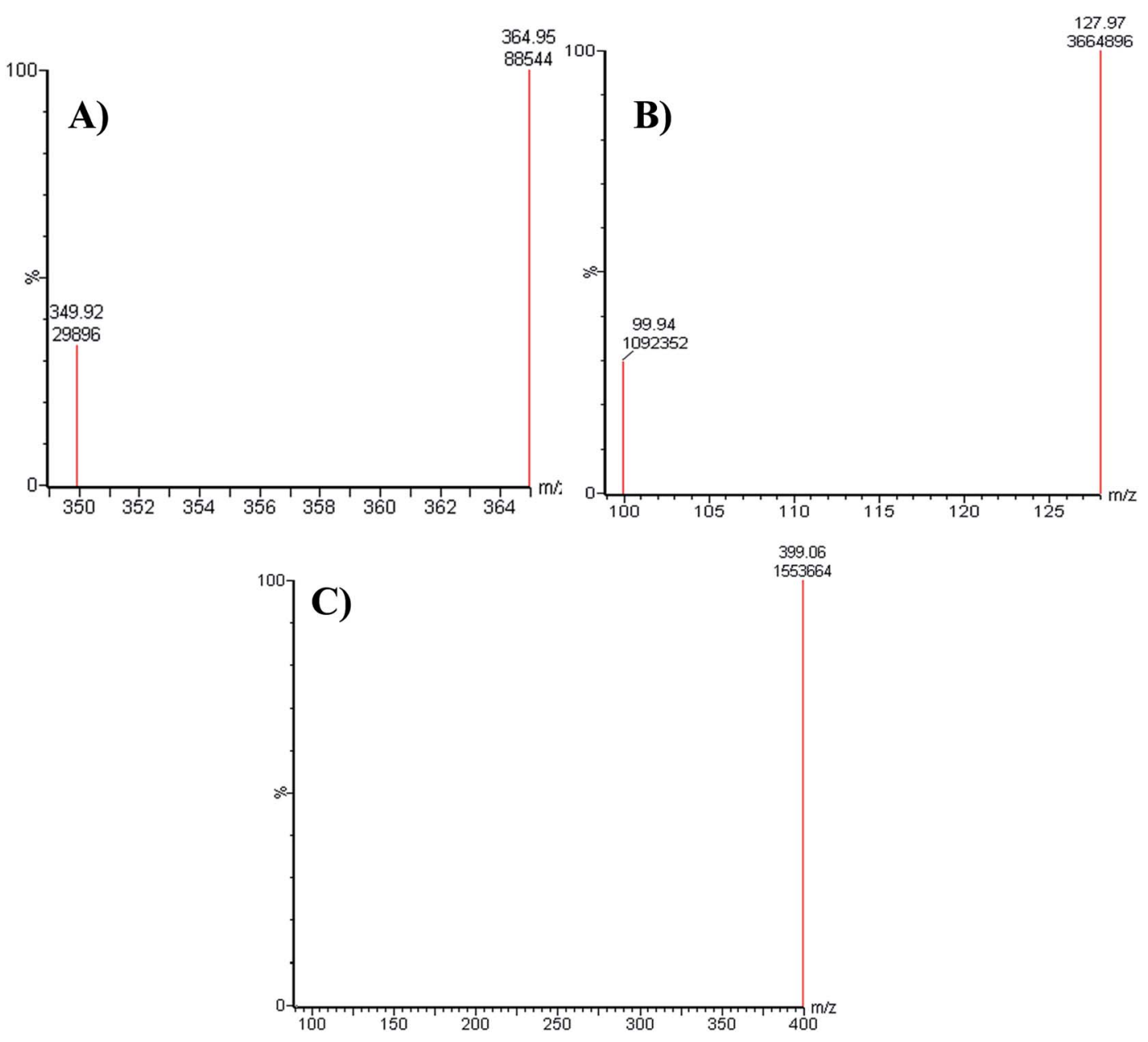

Fig. 2 MRM mass spectrum transitions of LPB (A), FTB (B) and MST (C). 

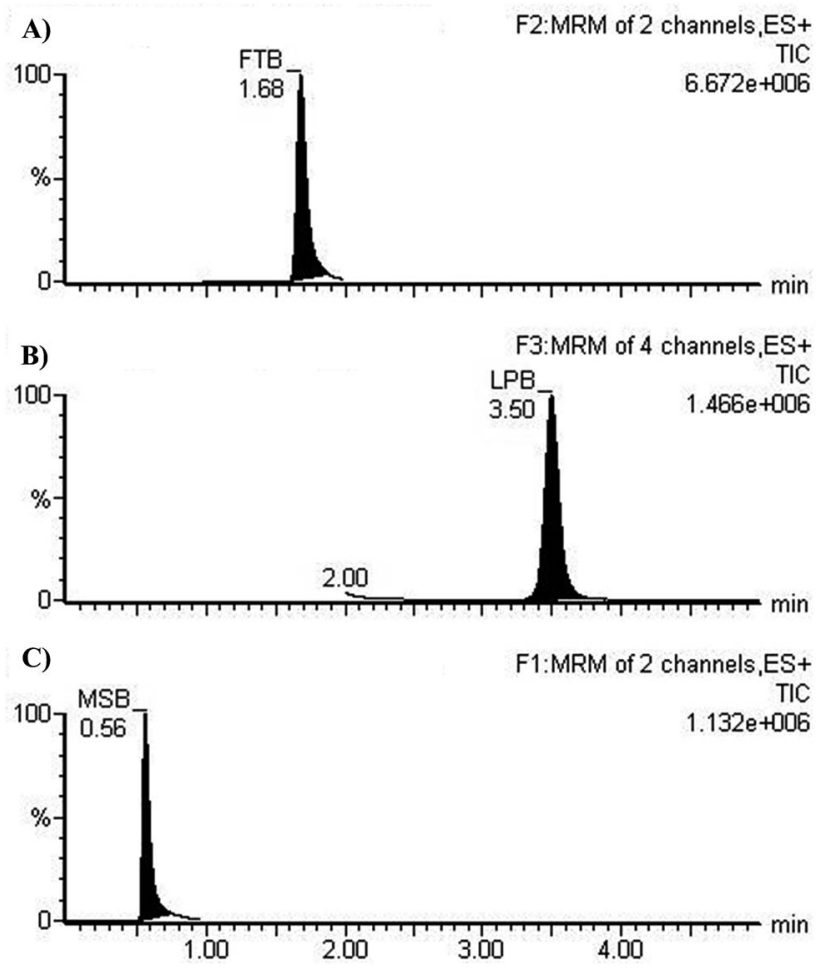

Fig. 3 MRM chromatograms of (A) FTB at $1.68 \mathrm{~min}$, (B) LPB at $3.5 \mathrm{~min}$ and (C) MST at 0.56 min.

\section{Experimental}

\subsection{Chemicals and reagents}

All reagents and solvents were of analytical grade. Lapatinib was purchased from Med Chem Express (USA). Foretinib (FTB) and masitinib (MST) were purchased from LC Laboratories (USA). Acetonitrile (ACN), Rat liver microsomes (RLMs), and formic acid $(\mathrm{HCOOH})$ were purchased from Sigma-Aldrich (USA). HPLC-grade water was obtained from the Milli-Q plus purification system (Millipore, USA).

\subsection{Liquid chromatography tandem mass spectrometry methodology}

An Acquity UPLC [model code (UPH) and serial number (H10UPH)] was used chromatographic separation of RLMs incubates while Acquity TQD MS [model code (TQD) and serial number (QBB1203)] was used for mass analysis of eluted analytes peaks. MST was selected as the IS in the FTB and LPB analysis as the same method of extraction could be applied for both MST, FTB and LPB. MST is considered a TKIs and are not reported to be administered together with either FTB or LPB to the same patient at the same time. Fragmentation pattern for the analytes were explained in Scheme 1. All LC-MS/MS parameters were adjusted for efficient and fast separation for tested analytes (Table 1). Multiple reaction monitoring (MRM) detection mode was used in mass analysis of LPB, FTB and IS (Scheme 1).

\subsection{Standard solutions of FTB and LPB}

FTB, LPB and MST are dimethyl sulfoxide soluble. Stock of FTB and LPB were prepared $\left(2 \mathrm{mg} \mathrm{mL}^{-1}\right)$ followed by ten times dilution with mobile phase to prepare FTB S1 $\left(200 \mu \mathrm{g} \mathrm{mL}^{-1}\right)$ and LPB S1 $\left(200 \mu \mathrm{g} \mathrm{mL}^{-1}\right)$, which was then further diluted ten times with mobile phase to prepare FTB S2 $\left(20 \mu \mathrm{g} \mathrm{mL}^{-1}\right)$ and LPB S2 $\left(20 \mu \mathrm{g} \mathrm{mL}{ }^{-1}\right)$. Stock of masitinib solution $\left(100 \mu \mathrm{g} \mathrm{mL}{ }^{-1}\right)$ was prepared in dimethyl sulfoxide and then further diluted fifty times with the mobile phase to prepare working MST S3 (2 $\mu \mathrm{g}$ $\left.\mathrm{mL}^{-1}\right)$.

\subsection{Preparation of calibration standards}

LPB S2 $\left(20 \mu \mathrm{g} \mathrm{mL}^{-1}\right)$ and FTB S2 $\left(20 \mu \mathrm{g} \mathrm{mL}{ }^{-1}\right)$ were diluted with specific RLMs matrix ( $40 \mu \mathrm{L}$ in $1 \mathrm{~mL}$ of phosphate buffer) to generate twelve calibration standards: 5, 10, 15, 30, 50, 75, 100,

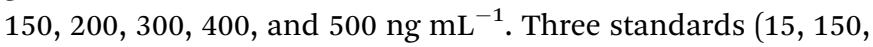

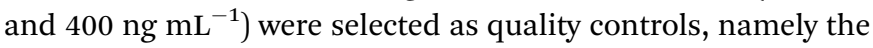
low quality control (LQC), medium quality control (MQC), and

Table 2 Data of LPB and FTB back-calculated concentration of the calibration levels from RLMs matrix

\begin{tabular}{|c|c|c|c|c|c|c|c|c|}
\hline \multirow{2}{*}{$\begin{array}{l}\text { Nominal concentrations } \\
\text { in } \mathrm{ng} \mathrm{mL}\end{array}$} & \multicolumn{4}{|l|}{ LPB } & \multicolumn{4}{|l|}{ FTB } \\
\hline & $\operatorname{Mean}^{a}$ & SD & $\mathrm{RSD} \%$ & Accuracy\% & $\operatorname{Mean}^{a}$ & $\mathrm{SD}$ & $\mathrm{RSD} \%$ & Accuracy\% \\
\hline 5 & 4.81 & 0.16 & 3.39 & 96.19 & 4.96 & 0.22 & 4.48 & 99.30 \\
\hline 10 & 9.51 & 0.29 & 3.06 & 95.12 & 10.33 & 0.51 & 4.93 & 103.26 \\
\hline 15 & 15.01 & 0.59 & 3.96 & 100.04 & 15.6 & 0.26 & 1.68 & 104.03 \\
\hline 30 & 30.39 & 1.04 & 3.41 & 101.29 & 29.7 & 0.83 & 2.79 & 99.00 \\
\hline 50 & 49.58 & 0.39 & 0.78 & 99.16 & 49.33 & 1.96 & 3.97 & 98.65 \\
\hline 75 & 74.83 & 0.55 & 0.74 & 99.78 & 73.51 & 2.31 & 3.14 & 98.01 \\
\hline 100 & 100.57 & 1.16 & 1.15 & 100.57 & 97.69 & 3.05 & 3.12 & 97.69 \\
\hline 150 & 147.41 & 5.73 & 3.88 & 98.27 & 147.77 & 3.51 & 2.38 & 98.51 \\
\hline 200 & 203.89 & 2.95 & 1.44 & 101.95 & 196.66 & 6.48 & 3.29 & 98.33 \\
\hline 300 & 301.54 & 1.75 & 0.58 & 100.51 & 300.88 & 8.68 & 2.89 & 100.29 \\
\hline 400 & 396.32 & 1.42 & 0.36 & 99.08 & 402.22 & 3.83 & 0.95 & 100.56 \\
\hline 500 & 501.25 & 2.39 & 0.48 & 100.25 & 508.61 & 17.13 & 3.37 & 101.72 \\
\hline$\%$ recovery & & & & 99.35 & & & & 99.95 \\
\hline SD & & & & 2 & & & & 2.08 \\
\hline
\end{tabular}


Table 3 Precision and accuracy (intra-day and inter-day) of the developed methods

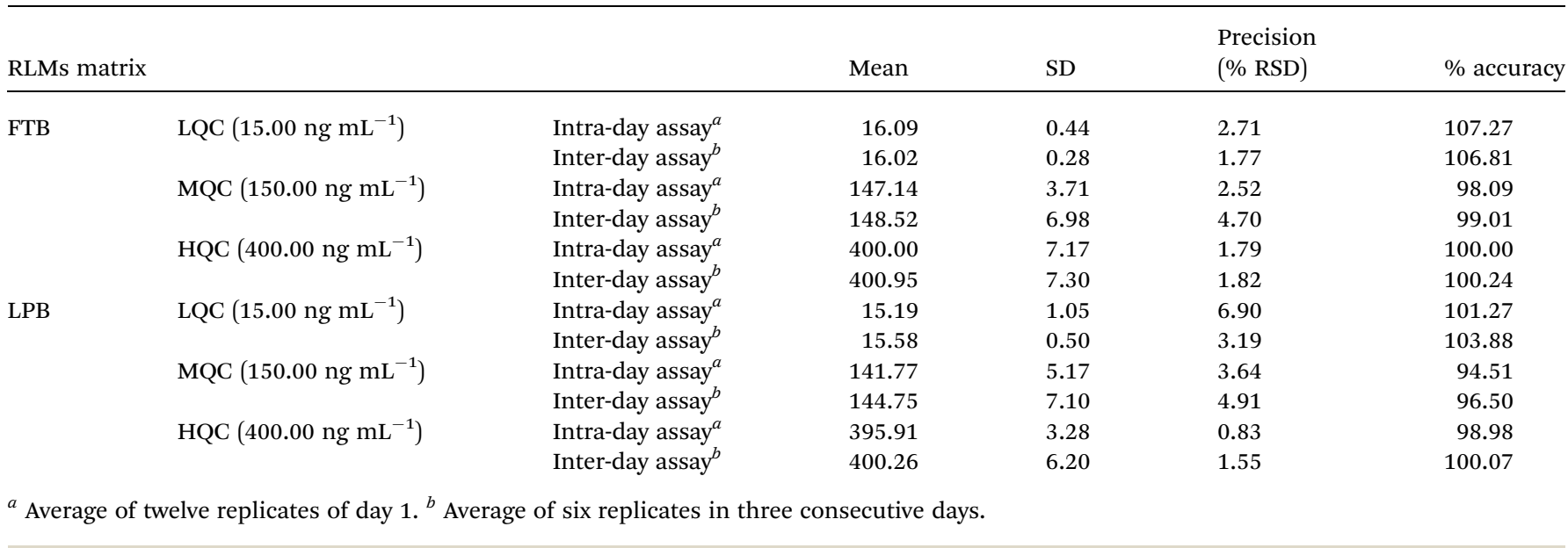

high quality control (HQC), respectively. Fifty microliters of MST S3 was added to $1 \mathrm{~mL}$ of each calibration standard.

\subsection{FTB and LPB extraction from RLMs matrix}

Analytes extractions were done using ACN protein precipitation, a standard technique for metabolic stability experiments. ${ }^{\mathbf{1 4 - 1 7}}$ ACN was used for precipitation followed by centrifugation (14000 rpm, $12 \mathrm{~min}$ at $4{ }^{\circ} \mathrm{C}$ ) then supernatants were filtered using syringe filters $(0.22 \mu \mathrm{m}$ pore size). Filtered samples were transferred to $1.5 \mathrm{~mL}$ vials. Five $\mu \mathrm{L}$ of each sample was then injected into the LC-MS/MS for analysis. Similarly, blank samples were prepared by using the stated phosphate buffer without RLMs matrix to confirm that RLMs components did not interfere with the elution time of analytes. Two calibration curve were established by plotting the peak area ratio of LPB to MST ( $y$ axis) against the nominal values ( $x$ axis) and the peak area ratio of FTB to MST ( $y$ axis) against the nominal values ( $x$ axis). A linear regression equation was used to validate the linearity of the established method. Slope, intercept, and coefficient of determination $\left(r^{2}\right)$ values were computed.

\subsection{Method validation}

Validation parameters of the established LC-MS/MS method were described in more detail previously ${ }^{\mathbf{1 5 , 1 8 , 1 9}}$ that included assay recovery, sensitivity, specificity, linearity, reproducibility, limit of detection (LOD) and limit of quantification (LOQ). The least squares statistical method was used to calculate the calibration curve equations $(y=a x+b)$. The linear fit was verified using the $R^{2}$ value, which was linear in the range from $2-500 \mathrm{ng} \mathrm{mL}^{-1}$.

\subsection{Metabolic stability of LPB and FTB}

The metabolic stability study for LPB and FTB were done by evaluating the decrease in LPB and FTB concentrations after incubation with RLMs. One $\mu \mathrm{M}$ FTB and $1 \mu \mathrm{M}$ LPB were incubated with RLMs (1 mg protein) in triplicate. The metabolic reaction medium was phosphate buffer $(\mathrm{pH} 7.4)$ that contains $3.3 \mathrm{mM} \mathrm{MgCl}_{2}$. The metabolic mixture was preincubated at $37{ }^{\circ} \mathrm{C}$ water bath for temperature conditioning for $10 \mathrm{~min}$. NADPH ( $1 \mathrm{mM})$ was used to initiate the metabolic reaction while $2 \mathrm{~mL}$ ACN was used to terminate it at specific time intervals $(0,2.5,5,7.5,10,15,20,40$, and $50 \mathrm{~min})$. The metabolic stability curve for FTB and LPB were then created. The same metabolic reaction was repeated for each analyte (LPB and FTB) in a separate experiment to assess the drug metabolic stability alone or in a mixture of both.

\section{Results and discussion}

\subsection{HPLC-MS/MS methodology}

All chromatographic parameters including the mobile phase $\mathrm{pH}$, mobile phase constituents, and $\mathrm{C}_{18}$ column were adjusted. The aqueous mobile phase part pH $\left(10 \mathrm{mM} \mathrm{NH}_{4}\right.$ $\mathrm{COOH}$ ) was adjusted to 4.2 with formic acid as above this

Table 4 Recovery of QC samples in RLMs matrix

\begin{tabular}{|c|c|c|c|c|c|c|}
\hline \multicolumn{7}{|c|}{ RLMs matrix } \\
\hline FTB & & & LPB & & & \\
\hline $\begin{array}{l}\text { Nominal concentration (ng } \\
\mathrm{mL}^{-1} \text { ) }\end{array}$ & $15 \mathrm{ng} \mathrm{mL}^{-1}$ & $150 \mathrm{ng} \mathrm{mL} \mathrm{L}^{-1}$ & $400 \mathrm{ng} \mathrm{mL} \mathrm{L}^{-1}$ & $15 \mathrm{ng} \mathrm{mL}^{-1}$ & $150 \mathrm{ng} \mathrm{mL}{ }^{-1}$ & $400 \mathrm{ng} \mathrm{mL} \mathrm{L}^{-1}$ \\
\hline Recovery (\%) & 107.23 & 98.15 & 99.85 & 95.97 & 99.25 & 100.48 \\
\hline SD & 0.68 & 5.78 & 11.19 & 0.67 & 1.84 & 2.47 \\
\hline Precision (RSD\%) & 4.23 & 3.93 & 2.8 & 4.66 & 1.24 & 0.62 \\
\hline
\end{tabular}

${ }^{a}$ Average of six replicates. 
Table 5 Stability data of FTB and LPB in RLMs matrix under different conditions

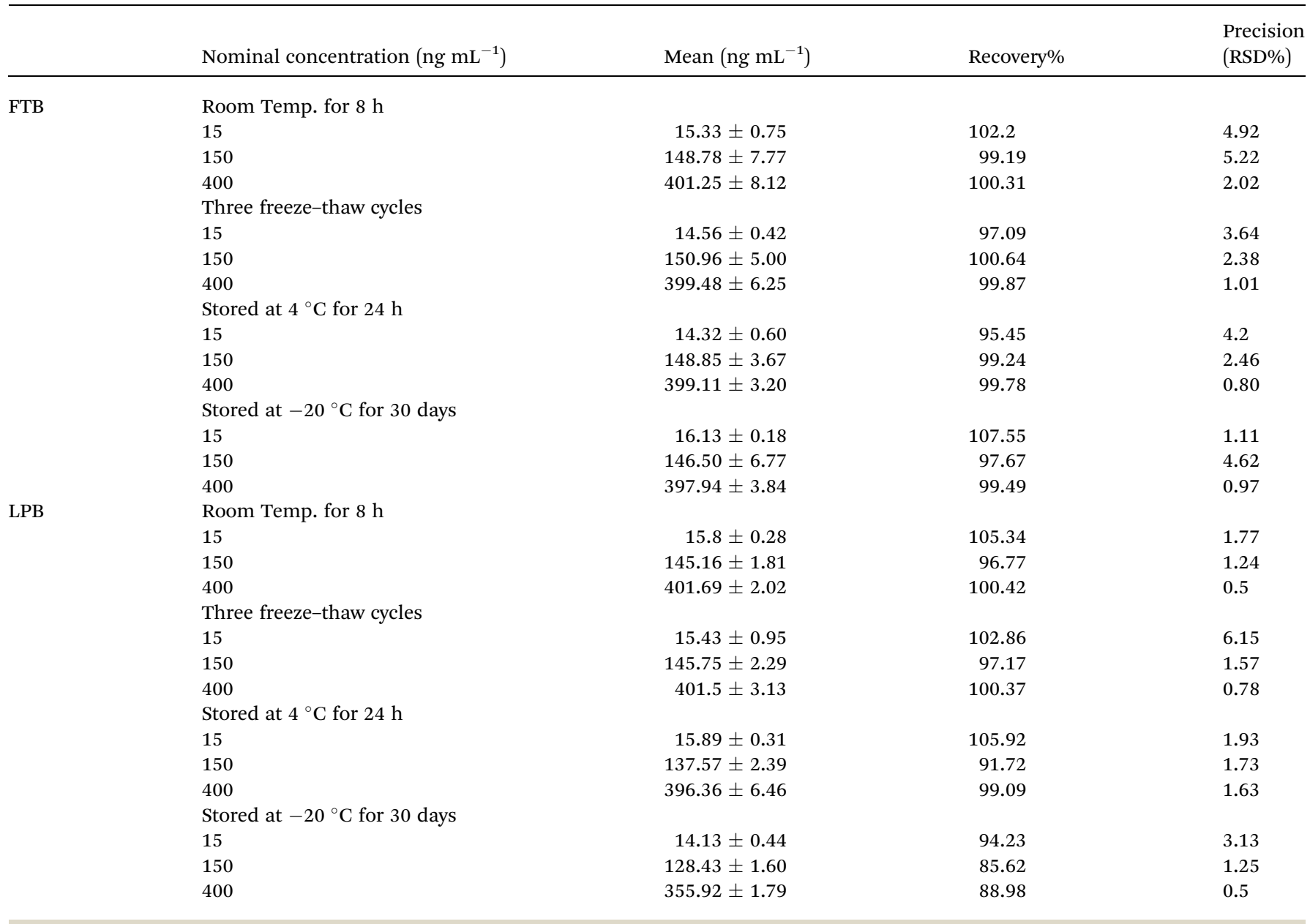

value caused unnecessary increase in elution time and peak tailing. The aqueous to organic (ACN) ratio parts of the mobile phase was optimized at $45 \%: 55 \%$ as ACN increase generated overlapped chromatographic peaks with bad resolution and ACN decrease resulted in higher elution times. Various types of stationary phases (e.g., HILIC columns) were checked, but LPB, FTB and MST were not separated. The perfect results were attained using $\mathrm{C}_{18}$ columns. The MRM mode of the mass analyzer was utilized for LPB and FTB ions estimation to avoid potential interference from the RLM matrix constituents and increase the sensitivity of the established LC-MS/MS method (Fig. 2).

The chromatographic resolution of LPB, FTB and MST were attained in 5 min with good resolution (Fig. 3).

\subsection{Method validation of the developed LC-MS/MS method}

3.2.1. Specificity. MRM chromatograms reveal good resolution of the LPB, FTB and IS peaks and the absence of peaks
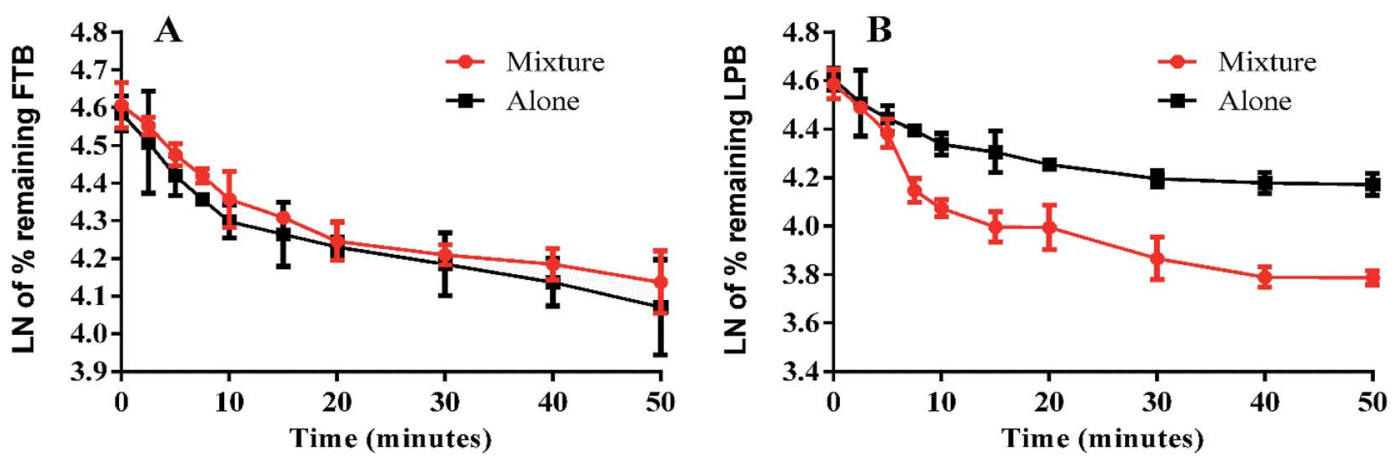

Fig. 4 Metabolic stability curve of FTB alone or in a mixture with LPB (A). Metabolic stability curve of LPB alone or in a mixture with FTB (B). 
Table 6 Metabolic stability parameters for FTB and LPB incubations with RLMs

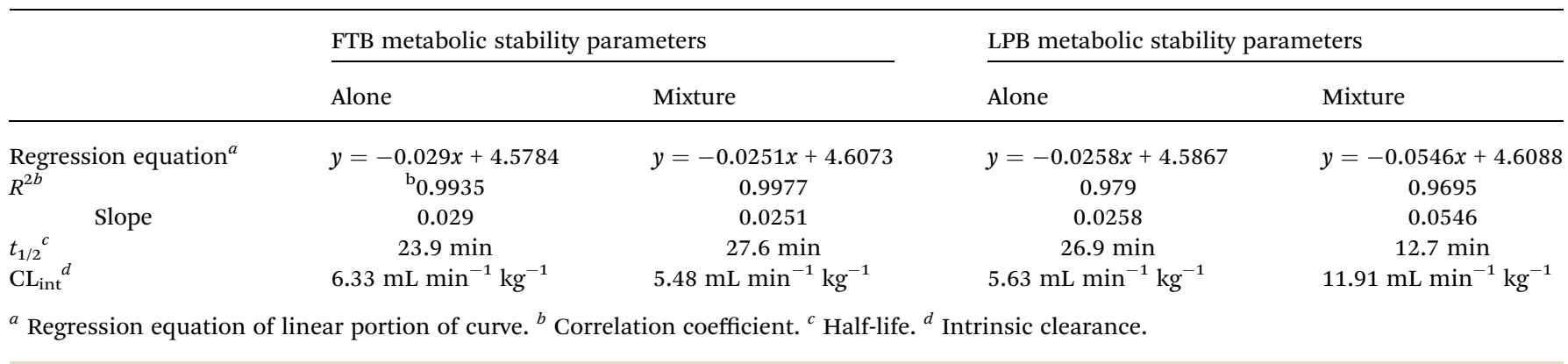

with the blank RLM matrix at their elution times, which approves the specificity of the established methodology (Fig. 3).

3.2.2. Sensitivity and linearity. The regression line linear range and correlation coefficient $\left(r^{2}\right)$ for the developed chro-

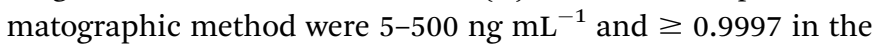
RLM matrix, respectively. The FTB calibration curve regression equation was $y=0.01925 x+0.2236$. LOD and LOQ for FTB calibration curve were equal to 1.2 and $3.64 \mathrm{ng} \mathrm{mL} \mathrm{m}^{-1}$, respectively. The LPB calibration curve regression equation was $y=$ $0.004721 x+0.03558$. LOD and LOQ for LPB calibration curve were equal to 2.66 and $8.051 \mathrm{ng} \mathrm{mL}{ }^{-1}$, respectively. The RSD values of six replicates for each standard level in the calibration curve were less than $4.93 \%$ and $3.96 \%$ (FTB and LPB) in the RLM matrix (Table 2). Back calculations of the fifteen standards of LPB in the RLM matrix (calibration and QC standards) approved the performance of the developed methodology.

3.2.3. Precision and accuracy. The intra- and inter-day accuracy and precision values are acceptable according to International Council for Harmonisation ( $\mathrm{ICH}$ ) guidelines (Table 3$)^{\mathbf{2 0 2} 21}$ as they are ranged from 0.36 to $3.96 \%$ and 95.12 to $101.95 \%$ for LPB, respectively, and ranged from 0.95 to $4.93 \%$ and 97.69 to $104.03 \%$ for FTB, respectively (Table 3 ).

3.2.4. Matrix effects and extraction recovery. Table 4 shows the recovery percentages of the quality controls when computing FTB and LPB concentration with the RLMs matrix. The FTB and LPB recoveries were $101.7 \pm 4.8 \%, 98.6 \pm 2.3 \%$, respectively in the RLMs matrix. The RLMs matrix effect absence was verified by analyzing six various batches of RLMs matrixes form six different rats. These batches (named set 1) were extracted then spiked with FTB and LPB LQCs (15 ng $\mathrm{mL}^{-1}$ ) and MST (IS). Set 2 batches were prepared in the same way utilizing the mobile phase instead of the RLMs matrix. Thus, the matrix effect was computed using the next equation:

$$
\text { Matrix effect }=\text { mean peak area ratio } \frac{\text { set } 1}{\text { set } 2} \times 100
$$

The tested RLM matrix that contained FTB and LPB had a matrix effect of $99.7 \pm 4.8 \%$ and $97.9 \pm 3.6 \%$, respectively. Accordingly, these outcomes approved that there is very low influence of the RLM matrix on FTB, LPB and MST (IS) ionization.

3.2.5. Stability. FTB and LPB stabilities of in RLMs matrix were tested under all laboratory conditions that may have been subjected before drug analysis. FTB and LPB exhibited good stability in RLMs matrix following storage at $-20{ }^{\circ} \mathrm{C}$ for 30 days. Measured values were ranged from 95.45 to $107.6 \%$ for FTB and from 85.6 to $105.9 \%$ for LPB. FTB and LPB stability data is mentioned in Table 5. There was no noticeable degradation of analytes under the examined conditions.

\subsection{Metabolic stability}

The LPB and FTB concentrations in the RLMs matrix were computed by substitution of the peak area ratios in the linear calibration curve regression equation. Metabolic stability curves were drawn for FTB and LPB alone or in a mixture (Fig. 4). The linear portion of the established curve was used to compute in vitro $t_{1 / 2} \cdot{ }^{22}$ The regression equations for this linear region were for FTB (alone: $y=-0.029 x+4.5784$ with $R^{2}=0.994$, mix: $y=$ $-0.0251 x+4.6073$ with $R^{2}=0.998$ ) and for LPB (alone: $y=$ $-0.0258 x+4.5867$ with $R^{2}=0.979$, mix: $y=-0.0546 x+4.6088$ with $R^{2}=0.969$ ) (Table 6).

The slope for each regression equation was substituted in the next equations:

$$
\begin{gathered}
\text { In vitro } t_{1 / 2}=\ln 2 / \mathrm{slope} \\
\text { In vitro } t_{1 / 2}=0.693 / \mathrm{slope}
\end{gathered}
$$

The intrinsic clearance $\left(\mathrm{CL}_{\mathrm{int}}\right)$ of $\mathrm{LPB}$ and FTB were computed using the in vitro $t_{1 / 2} \operatorname{method}^{\mathbf{1 3}}$ as in the following equation:

$$
\begin{aligned}
\mathrm{CL}_{\text {int }}= & \frac{0.693}{\text { in vitro } t_{1 / 2}} \times \frac{\mathrm{mL} \text { incubation }}{\mathrm{mg} \text { microsomes }} \\
& \times \frac{45 \mathrm{mg} \text { microsome }}{\mathrm{g} \text { liver }} \times \frac{20 \mathrm{~g} \text { liver }}{\mathrm{kg} \text { per body weight }} \\
& \mathrm{CL}_{\text {int }}=\frac{0.693}{\text { in vitro } t_{1 / 2}} \times \frac{1.45}{112.5} \times \frac{20}{0.33}
\end{aligned}
$$

From these outcomes, FTB and LPB metabolic stabilities were characterized by a high $\mathrm{CL}_{\text {int }}\left(6.33\right.$ and $5.63 \mathrm{~mL} \mathrm{~min}{ }^{-1}$ $\mathrm{kg}^{-1}$ ) and a low in vitro $t_{1 / 2}(23.9$ and $26.9 \mathrm{~min})$. FTB metabolic rate is slightly decreased in combination with LPB, while LPB 
metabolic rate is greatly increased in combination with FTB (Table 6).

\section{Conclusions}

A validated LC-MS/MS methodology was developed for simultaneous estimation of FTB and LPB. The established method is highly sensitive, echo friendly (small volume of ACN), fast (run time $=5 \mathrm{~min}$.), accurate, and has high recovery. The metabolic stability of FTB and LPB were characterized by a high $\mathrm{CL}_{\text {int }}(6.33$ and $5.63 \mathrm{~mL} \mathrm{~min}^{-1} \mathrm{~kg}^{-1}$ ) and a low in vitro $t_{1 / 2}$ (23.9 and 26.9 min), which revealed a high clearance of FTB and LPB from the blood by the liver. This probably resulted in a low in vivo bioavailability that corroborated the low oral bioavailability previously reported and also indicated that FTB and LPB will not bioaccumulate after multiple doses. FTB metabolic rate is slightly decreased in combination with LPB, while LPB metabolic rate is greatly increased in combination with FTB. So dose recalculation should be considered when FTB and LPB are used in combination.

\section{Conflicts of interest}

There are no conflicts to declare.

\section{Authors' contributions}

MAA, MWA, HAA, and HWD designed the study. MAA, HAA, and AAA supervised the practical work. AHB, MAA and MWA performed the optimization and method validation studies. MWA wrote the first version of the manuscript. All authors approved and revised the final version of the manuscript.

\section{Abbreviations}

$\begin{array}{ll}\text { ACN } & \text { Acetonitrile } \\ \text { FTB } & \text { Foretinib } \\ \text { LPB } & \text { Lapatinib } \\ \text { CL }_{\text {int }} & \text { Intrinsic clearance } \\ \text { CID } & \text { Collision-induced dissociation } \\ \text { ESI } & \text { Electrospray ionization } \\ \text { LC-MS/ } & \text { Liquid chromatography tandem mass spectrometry } \\ \text { MS } & \\ \text { PI } & \text { Product ion } \\ \text { RLM } & \text { Rat liver microsomes } \\ \text { TKIs } & \text { Tyrosine kinase inhibitors } \\ t_{1 / 2} & \text { In vitro half-life } \\ \text { VEGFR } & \text { Vascular endothelial growth factor receptor-2 } \\ \text { MET } & \text { Mesenchymal-epithelial transition factor } \\ \text { MST } & \text { Masitinib } \\ \text { PI } & \text { Product ion } \\ \text { LOD } & \text { Limit of detection } \\ \text { LOQ } & \text { Limit of quantification } \\ \text { MRM } & \text { Multiple reaction monitoring }\end{array}$

\section{Acknowledgements}

The authors would like to extend their sincere appreciation to the Deanship of Scientific Research at the King Saud University for funding this work through the Research Group Project No. RG-1440-052.

\section{References}

1 S. R. Hubbard and J. H. Till, Ann. Rev. Biochem., 2000, 69, 373-398.

2 C. Natoli, B. Perrucci, F. Perrotti, L. Falchi and S. Iacobelli, Curr. Cancer Drug Targets, 2010, 10, 462-483.

3 J. Schlessinger, Cell, 2000, 103, 211-225.

4 C. Özvegy-Laczka, J. Cserepes, N. B. Elkind and B. Sarkadi, Drug Resist. Updates, 2005, 8, 15-26.

5 N. Steeghs, J. W. Nortier and H. Gelderblom, Ann. Surg. Oncol., 2007, 14, 942-953.

6 Y. Kataoka, T. Mukohara, H. Tomioka, Y. Funakoshi, N. Kiyota, Y. Fujiwara, M. Yashiro, K. Hirakawa, M. Hirai and H. Minami, Invest. New Drugs, 2012, 30, 1352-1360.

7 G. M. Higa and J. Abraham, Expert Rev. Anticancer Ther., 2007, 7, 1183-1192.

8 A. J. Tevaarwerk and J. M. Kolesar, Clin. Ther., 2009, 31, 23322348.

9 X. Wan, X. Zheng, X. Pang, Z. Zhang and Q. Zhang, Colloids Surf., B, 2015, 136, 817-827.

10 Z. Hartman, H. Zhao and Y. M. Agazie, Oncogene, 2013, 32, 4169-4180.

11 P. J. Medina and S. Goodin, Clin. Ther., 2008, 30, 1426-1447. 12 S. K. Chia, S. L. Ellard, M. Mates, S. Welch, C. Mihalcioiu, W. H. Miller, Jr., K. Gelmon, C. Lohrisch, V. Kumar, S. Taylor, L. Hagerman, R. Goodwin, T. Wang, S. Sakashita, M. S. Tsao, E. Eisenhauer and P. Bradbury, Breast Cancer Res., 2017, 19, 54.

13 P. Baranczewski, A. Stanczak, K. Sundberg, R. Svensson, A. Wallin, J. Jansson, P. Garberg and H. Postlind, Pharmacol. Rep., 2006, 58, 453-472.

14 M. W. Attwa, A. A. Kadi, H. W. Darwish, S. M. Amer and H. Alrabiah, Eur. J. Mass Spectrom., 2018, 24, 344-351.

15 A. A. Kadi, H. W. Darwish, M. W. Attwa and S. M. Amer, PLoS One, 2016, 11, e0164967.

16 H. W. Darwish, A. A. Kadi, M. W. Attwa and H. S. Almutairi, Clin. Chim. Acta, 2018, 480, 180-185.

17 A. A. Kadi, A. S. Abdelhameed, H. W. Darwish, M. W. Attwa and A. H. Bakheit, Trop. J. Pharm. Res., 2016, 15, 2683-2692.

18 S. M. Amer, A. A. Kadi, H. W. Darwish and M. W. Attwa, Chem. Cent. J., 2017, 11, 45.

19 A. A. Kadi, R. F. Angawi, M. W. Attwa, H. W. Darwish and A. S. Abdelhameed, J. Chem., 2013, 2013, 905704.

20 H. W. Darwish, A. A. Kadi, M. W. Attwa and H. S. Almutairi, Clin. Chim. Acta, 2018, 480, 180-185.

21 M. W. Attwa, A. A. Kadi, H. W. Darwish, S. M. Amer and H. Alrabiah, Eur. J. Mass Spectrom., 2018, 24, 344-351.

22 G. Caldwell and Z. Yan, Optimization in drug discovery: in vitro methods, Springer Science \& Business Media, 2014. 